\title{
6. Umgang mit eigenen Fehlern: CIRS
}

\author{
Manfred Lang
}

Bei jeder Tätigkeit, die Menschen ausführen, passieren Fehler. Dies gilt natürlich auch für den Bereich der Medizin. CIRS (Abk. für Critical Incident Reporting System) ist ein Instrument im Rahmen des Risikomanagements, um die Häufigkeit von Fehlern zu senken.

Entwickelt wurde CIRS in der Nuklear- und der Luftfahrtindustrie. Fehler in diesen Bereichen haben immer wieder verheerende Konsequenzen, weshalb man sich dort seit vielen Jahren intensiv mit der Fehleranalyse beschäftigt hat. Eine der wesentlichen Erkenntnisse ist, dass einem tatsächlich eingetretenen Fehler eine große Anzahl von Beinahefehlern vorausgeht. Ein Beinahefehler (sog. Critical Incident) führt zu keinem erkennbaren Schaden. Für sich allein betrachtet ist er in der Regel harmlos, in der Verkettung mit anderen Beinahefehlern oder ungünstigen Umständen führt er aber zu einem tatsächlichen Fehler oder Schadensereignis. Mit einem CIRS versucht man, eine möglichst große Anzahl von Beinahefehlern zu erfassen und deren Entstehung für die Zukunft zu verhindern, in der Annahme, die sich möglicherweise entwickelnden tatsächlichen Fehler ebenfalls zu reduzieren.

Diese grundsätzliche Betrachtungsweise ist auch auf die Medizin übertragbar.

Die Behandlung eines Patienten setzt sich aus einer großen Zahl komplexer Behandlungsschritte zusammen, die von verschiedenen Abteilungen und Berufsgruppen erbracht werden. In diesem komplexen Zusammenspiel sind gegenseitige Missverständnisse, Fehlinterpretationen, individuelle Unerfahrenheit, Materialfehler und dergleichen ständige Begleiter, erst recht unter steigendem Zeitdruck. Sie werden aber meistens durch unterschiedliche Überwachungsverfahren (z.B. Visiten, „Vier-Augen-Prinzip“) kompensiert. Erst in einer spezifischen, in der 
Regel als unvorhergesehen eingeschätzten Konstellation dieser Faktoren (Beinahefehler) kommt es zu einem tatsächlichen Schadensfall am Patienten.

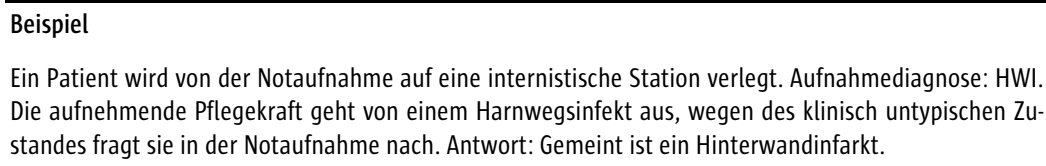

Für ein funktionierendes CIRS muss eine Reihe von Voraussetzungen erfüllt sein. Ganz wichtig ist die Unterscheidung von stattgehabten, tatsächlichen Fehlern und Beinahefehlern. Tatsächliche Behandlungsfehler werden einer Schadensfallanalyse unterzogen und die Schuldfrage ggf. in einer juristischen Auseinandersetzung geklärt. Die Patienten- und Behandlungsdokumente können ggf. von der Staatsanwaltschaft beschlagnahmt werden. Definitionsgemäß ist durch Beinahefehler noch kein Schaden eingetreten. Um auf jeden Fall die meldende Person vor Ermittlungen zu schützen, muss die Identität des Meldenden anonym bleiben. Dazu werden in den existierenden CIRS verschiedene Rechtskonstruktionen genutzt, z.B. das Presserecht. Für das einzelne Krankenhaus gibt es verschiedene Möglichkeiten, wie die Anonymität der Meldenden sichergestellt werden kann. Natürlich ist die Anonymität nicht nur als Schutz vor möglichen staatsanwaltschaftlichen Ermittlungen wichtig, sondern auch als Schutz vor Sanktionen durch Vorgesetzte, da CIRS-Meldungen oftmals unbequeme Konsequenzen bedingen. Damit ist eine weitere wichtige Voraussetzung für CIRS erkennbar. Die oberste Krankenhausleitung und damit alle Führungskräfte müssen CIRS nicht nur tolerieren, sondern aktiv unterstützen!

Weiterhin müssen eine oder mehrere niederschwellige Meldemöglichkeiten von Beinahefehlern für alle Berufsgruppen geschaffen werden. Grundsätzlich kann dies in Papierform oder IT-gestützt erfolgen. Die entsprechenden Formulare oder die Software-Ausstattung sind zu erstellen.

\section{Wichtig ist die Freiwilligkeit der Meldung.}

Mit der Auswertung der eingehenden Meldungen beschäftigt sich eine regelmäßig tagende CIRS-Gruppe. Vor allem für die Bewertung der eingehenden Meldungen sollte dieser Personenkreis die verschiedenen Arbeitsabläufe im Krankenhaus gut kennen. Dies gilt sowohl für die formalen Abläufe als auch für die medizinischen und pflegerischen Behandlungspfade. Der angesprochene Personenkreis sollte die Meldungen unbedingt in kurzfristigen Intervallen sichten, um auch auf akut bedrohliche Situationen angemessen schnell reagieren zu können. Der angesprochenen CIRS-Cruppe obliegt als weitere wichtige Aufgabe die Entscheidung darüber, wie die gemeldeten kritischen Situationen in Zukunft zu verhindern sind. Abhängig von der Meldung kann dies von der kurzfristig möglichen Behebung einer technischen Fehlfunktion bis zur Veränderung langjährig gewohnter, komplexer Abläufe im Krankenhaus reichen. Dafür sind die Konse- 
quenzen für die Mitarbeiter oft mehrerer Abteilungen aus unterschiedlichen Berufsgruppen zu bedenken. Das ist in der Regel nur unter Einbeziehung der ärztlichen und pflegerischen Leitungsebenen zu erreichen. Darüber hinaus muss die vollzogene Änderung kontrolliert, d.h. als funktionierend eingeschätzt werden.

\section{Eine entsprechende Rückmeldung erhält auch die meldende Person.}

Der oben bereits angesprochenen Leitungsebene des Krankenhauses obliegt die Sicherstellung der erforderlichen personellen und materiellen Ressourcen für ein CIRS. Die Mitglieder der CIRS-Gruppe beispielsweise sind in der Regel mit der Wahrnehmung ihrer normalen täglichen Aufgaben voll ausgelastet. Zur Sicherstellung der CIRS-Routine müssen sie freigestellt werden, was meist nur durch geplante Freistellung im Dienstplan (plus Vertreterregelung) gewährleistet werden kann.

Aus der Erfahrung, dass auch andere Krankenhäuser von gemeldeten CIRS-Fällen profitieren können, haben sich in den letzten Jahren nationale und internationale Netzwerke entwickelt, die die Überprüfung von relevanten Fällen für das eigene Krankenhaus ermöglichen. Die Netzwerke bieten unter anderem anonyme Bearbeitung und Auswertungen von CIRS-Fällen an. Mittlerweile existieren solche Netzwerke auch im Bereich der niedergelassenen Ärzte in den Praxen. Als Beispiel sei das „Netzwerk CIRS-Berlin“ der Ärztekammer Berlin genannt. Außerdem werden CIRS-Fälle regelmäßig in Fachzeitschriften offen erörtert.

\section{Probleme}

Die Erfüllung dieser Voraussetzungen für ein lebendiges CIRS ist für die meisten Krankenhäuser eine sehr große Herausforderung. Nach der ersten Einführung stellen viele Krankenhäuser fest, dass CIRS tatsächlich einer kontinuierlichen, offensiven Begleitung bedarf. Probleme wie mangelnde Beteiligung, fehlende Bereitschaft höherer Hierarchieebenen, sich vorgeschlagenen Lösungen unterzuordnen, oder fehlende Rückmeldung an die Meldenden können nur durch hartnäckiges und engagiertes Eintreten der Protagonisten schrittweise einer grundlegenden Lösung nähergebracht werden. Bei mangelnder Unterstützung läuft CIRS Gefahr, zu einer „Feigenblatt“-Funktion zu degenerieren und somit seine Möglichkeiten nicht ausschöpfen zu können.

Das große Ziel ist es, nicht nur eine angstfreie, vor Sanktionen geschützte Atmosphäre im Krankenhaus zu schaffen, sondern die Erkenntnis zu etablieren, dass mit CIRS ein offensives Instrument zur Verbesserung der Patientenversorgung zur Verfügung steht. Letztlich geht es um das Erreichen einer offeneren Kommunikationskultur im Krankenhaus über die verschiedenen Berufsgruppen- und Abteilungsgrenzen hinweg. Da erfahrungsgemäß der bei weitem größte Teil der Meldungen aus dem Pflegebereich kommt, kommt den Pflegekräften eine große Bedeutung zu. Der in den meisten deutschen Krankenhäusern seit Jahrzehnten gepflegte defensive Umgang mit kritischen Themen muss in den nächsten Jah- 
6 Umgang mit eigenen Fehlern: CIRS

ren schrittweise in eine diesbezügliche offensive Kommunikationskultur verändert werden. 